\title{
Differential Equations in Stability Analysis of Ferrofluids
}

\author{
Dr.R.Vasanthkumari ${ }^{1}$, A.Selvaraj ${ }^{2}$ \\ ${ }^{1}$ Principal ,Kasthurba College For Women, Villianur, Pudhuchery-605110, India. \\ ${ }^{2}$ Research Scholar Category-C, Manonmaniam Sundaranar University, Thirunelveli India.
}

\begin{abstract}
The application of differential equations towards stability analysis of ferrofluids is analysis both in porous medium and nonporous medium and a comparative analysis is made. Weakly non- linear analysis is carried out. A mathematics model of the differential equations employed is presented. The non-dimensional thermal Rayleigh number $R a$ and magnetic Rayleigh number Rm are analysed with allowable range of parameter.
\end{abstract}

Key Words: Ferrofluids, Mathematical Model, Non Porous Medium, Porous Medium, Weakly Non Linear Equations.

\section{Introduction}

Heat transfer through Ferrofluids subjected to strong magnetic fields, has notable application in technology of generator and motors. The Ferrofluids have a distinct advantage as it can be effectively used as a coolant in transfer is apt in armature of generators and motors, which rotates with constant angular velocity

Heat transfer though Ferrofluids, subjected to very high magnetic fields, finds remarkable applications in transformer technology. In an attempt to replace solid core by liquid core, the Ferrofluids have an added advantage as it can also be effectively used as a transformer. The method of formation of Ferrofluids was evolved in the early of mid -1960s, due to the availability of colloidal magnetic fluids (Ferro fluids) many uses of these fascinating liquids have been identified, which are concerned with the remove positioning and control of the magnetic fluid using magnetic force fields.

\section{Review Of Literature:}

(Ferrofluids are highly applied in lubrication, printing, and vacuum technology The novel zero-leakage rotary-shaft seals are used in computer disk drives [Bailey(1983)]. An authoritative introduction to this fascinating subject has been discussed in detail in the celebrated monograph by Rosensweig (1985). This monograph reviews several applications of heat transfer through Ferrofluids and one such phenomenon is enhanced convective cooling having a temperature-dependent magnetic moment due to magnetization of the fluid. This magnetization, in general, is a function of the magnetic field, temperature and density of a the fluid. Any variation of these quantities can induce a change of body force distribution in the fluid. This leads to convection is Ferrofluids. Is the presence of magnetic field gradient. This mechanism is known as Ferro convection, which is similar to Benard convection [chadrasekarhar1981].(convective instability of uniform vertical magnetic field has been considered by Finlayson(1970)).(Sunil and Mahajan (Sunil and Mahajan 2008) studied the non-linear stability analysis of magnetized Ferro fluid, with internal angular momentum, heated from bellow saturating a porous medium of high permeability via generalized energy method using Brinkman model. Attia(Attia 2009) studied the effect of the porosity of the medium on velocity components and temperature for the steady flow and heat transfer. Attia (Attia 2008) investigated steady laminar flow of an in compressible viscous non- Newtonian fluid due to the uniform rotation of porous disk of infinite extent in porous medium with heat transfer differential equations are effectively used in the stability analysis of Ferro fluids.

\section{Mathematical Formulation:}

An infinitely spread thin layer of Ferrofluids contained between two rigid boundaries heated from below is studied. The fluid is assumed to satisfy the Oberbeck-Boussinesq approximation. The magnetisation $\mathrm{M}$ of the Ferrofluids is assumed to be parallel to the local magnetic field $\mathrm{H}$. The present analysis deals with the special case of very strong magnetic fields

\section{NON POROUS MEDIUM}

$\nabla \cdot q=0$

The governing equations are

$$
\frac{\partial q}{\partial t}+(q \cdot \nabla) q=-\frac{\nabla p}{\rho_{0}}+\frac{\rho g}{\rho_{0}}+\nabla \cdot(B H)+v \nabla^{2} q
$$


Where $\mathrm{P}$ is the pressure and $\mathrm{q}=(\mathrm{u}, \mathrm{v}, \mathrm{w})$ the velocity, $\mathrm{g}=(\mathrm{o}, \mathrm{o},-\mathrm{g})$ the acceleration due to gravity, $\mathrm{B}$ the magnetic induction, $\mathrm{H}$ the magnetic field, $\rho$ the density, $\rho_{0}$ the density at $\mathrm{T}=\mathrm{T}_{0}$ and $\mathrm{V}$ the kinematic viscosity.

$$
\text { Energy equation is } \frac{\partial T}{\partial t}+(q . \nabla) T=k . \nabla^{2} T
$$

Usual magnetic equation of state and other related equation are considered where $\mathrm{T}$ is the temperature and $\mathrm{K}$ is the thermal conductivity where $\mathrm{M}$ is the magnetization, $\mathrm{M}$ is the constant mean value of the magnetisation and $\mathrm{K}$ is the Pyro magnetic coefficient.

For strong magnetic applied field in the Ferrofluids,

$$
M=\bar{M}+K \beta d \theta \hat{K}
$$

When the fluid is assumed to be non-conductive

$$
\nabla \times H=0
$$

$$
H=\bar{H}+K \beta d \nabla \Phi \text { (For magnetic field in the Ferro fluids) }
$$

Where $\Phi$ is the magnetic potential. Following stability analysis procedure,

The non-dimensional governing equations are

$$
\begin{gathered}
\nabla \cdot q=0 \\
-p r^{-1}\left[\frac{\partial}{\partial t}+(q \cdot \nabla)\right] q+R_{M} \theta \nabla \Phi_{Z}=\nabla^{2} q+\left(R_{a}+R_{M}\right) \theta \hat{k}-R_{M} \Phi_{Z} \hat{k}-\nabla p \\
\left(\frac{\partial}{\partial t}+(q . \nabla) \theta\right)=\nabla^{2} \theta+w
\end{gathered}
$$

$R_{M}=\frac{\mu_{0} K^{2} \beta^{2} d^{4}}{k v \rho_{0}}$ is the magnetic

$$
\text { and } \quad \nabla^{2} \Phi-\theta_{z}=0
$$

Rayleigh number and $p_{r}=\frac{v}{k}$ is the prandt 1 number.

The linear stability analysis carried out,$\quad \mathrm{f}=\mathrm{f}(\mathrm{z}) \exp (\mathrm{iax}+\sigma t)$

On eliminating pressure p, the non dimensional governing equations in $\Psi, \Theta$ and $\Phi$ are given by

$$
-p r^{-1} \sigma\left(D^{2}-a^{2}\right) \Psi+T a \Pi^{2} \Psi+\left(D^{2}-a^{2}\right)^{2} \Psi-i a R_{T}\left(\frac{1+R_{T}}{S}\right) \Theta+i a\left(\frac{R_{T}{ }^{2}}{S}\right) D \Phi=0
$$

$$
\begin{aligned}
& \text { where } R_{T}=\frac{g \alpha \beta d^{4}}{k v} \text { is the conventional thermal Rayleigh number } \\
& -\sigma \Theta-i a \Psi+\left(D^{2}-a^{2}\right) \Theta=0 \\
& -D \Theta+\left(D^{2}-a^{2}\right) \Phi=0
\end{aligned}
$$

The boundary condition for rigid, conducting boundaries on $\Psi$ and $\theta$ are given by

$$
\Psi=D \Psi=\Theta=0 \quad, \quad \text { for } \mathrm{z}= \pm 1 / 2
$$

And for non magnetic plates

$$
\left((D \pm a) \Phi_{z}= \pm \frac{1}{2}=0\right.
$$

Simplifying the Rayleigh number can be easily obtained as

$$
R_{T}=\frac{-h S+h\left[h^{2} S^{2}+4 h S\left(h^{3}+T a \Pi^{2}\right)\right]^{1 / 2}}{2 a^{2}}
$$

For weakly non linear system, finite amplitude technique adopted by Malkus and Veronis(1958) has been used retaining only first order terms. Dynamical variables $\mathrm{f}(\mathrm{x}, \mathrm{z}, \mathrm{t})$ are expanded in the following manner.

$$
\mathrm{f}(\mathrm{x}, \mathrm{z}, \mathrm{t})=\varepsilon 1 / 2\left[f_{11}(z, \tau) E+c c\right]+\varepsilon\left\{f_{02}(z, \tau)+\left[f_{22}(z, \tau) E^{2}+c c\right]\right\}+\varepsilon 3 / 2\left[f_{13}(z, \tau) E+c c\right]+\ldots
$$

Nusselt number is obtained as 


$$
N u=1+\left\{\frac{\left(R_{T}-R_{T c}\right)}{K_{R}}\right\}\left(D \Theta_{02}\right)_{z=1 / 2}
$$

Nu-1 measures the ratio of the heat transfer by convection to that by conduction wheras the Nusselt number $\mathrm{Nu}$ measure the ratio of the total heat transfer across a horizontal plane to the heat transfer by conduction alone

\section{POROUS MEDIUM}

Only the momentum equation is different from that of non porous medium

$$
\frac{\partial q}{\partial t}+(q . \nabla) q=-\frac{\nabla p}{\rho_{0}}+\frac{\rho g}{\rho_{0}}+\nabla \cdot(B H)+v \nabla^{2} q-\frac{v}{k_{0}} q
$$

Where $k_{0}$ is the permeability parameter. Follows similar procedure as in non porous medium we get the existence of porous medium inhibits convection. The effect is more when permeability of porous medium is small and reverse phenomenon will take place when permeability is large.

The thermal Rayleigh number $R_{T}$ is given by

$$
R_{T}=\frac{\sqrt{-h S \pm\left[S^{2}+4 S\left(h^{2}-\frac{1}{k_{0}}\right)\right]}}{2 a^{2}}
$$

And with weakly non linear analysis the Nusselt number is calculated as

$$
\mathrm{Nu}=1+\left\{\frac{\left(R_{T}-R_{T c}\right)}{K_{R}}\right\}\left(D \Theta_{02}\right)_{Z=1 / 2}(19)
$$

\begin{tabular}{|c|c|c|c|c|c|c|c|c|}
\hline \multirow{3}{*}{$\log s$} & \multicolumn{8}{|c|}{ Permeability Parameter $\mathrm{k}_{0}$} \\
\hline & \multicolumn{4}{|c|}{ Heated from below } & \multicolumn{4}{|c|}{ Heated from above } \\
\hline & $\mathrm{K}_{0=0} 0.01$ & $\mathrm{~K}_{0=0} 0.03$ & $\mathrm{~K}_{0=} 0.1$ & $\mathrm{~K}_{0=0} 0.3$ & $\mathrm{~K}_{0=0.01}$ & $\mathrm{~K}_{0=} 0.03$ & $\mathrm{~K}_{0=0.1}$ & $\mathrm{~K}_{0=0} 0.3$ \\
\hline-1 & 6.16 & 3.59 & 2.23 & 1.79 & -2.11 & -0.59 & 0.78 & 1.29 \\
\hline 0 & 2.64 & 1.98 & 1.73 & 1.62 & -0.29 & 0.54 & 1.28 & 1.46 \\
\hline 1 & 1.59 & 1.58 & 1.56 & 1.55 & 0.33 & 0.83 & 1.52 & 1.57 \\
\hline 2 & 0.78 & 1.35 & 1.48 & 1.52 & 0.62 & 1.03 & 1.52 & 1.57 \\
\hline 3 & 0.38 & 1.13 & 1.38 & 1.46 & 0.95 & 1.28 & 1.62 & 1.63 \\
\hline 4 & 0.28 & 0.81 & 1.39 & 1.39 & 1.44 & 1.63 & 1.79 & 1.79 \\
\hline
\end{tabular}

Table.1: variation of Nusselt number $\frac{(N u-1)}{\left(\frac{R_{T}}{R_{T c}}-1\right)}$ with the parameter $\mathrm{S}=\frac{R_{T}{ }^{2}}{R_{M}}$ for different permeability $k_{0}$

\section{IV . Table And Figure}

\section{FIFURE}

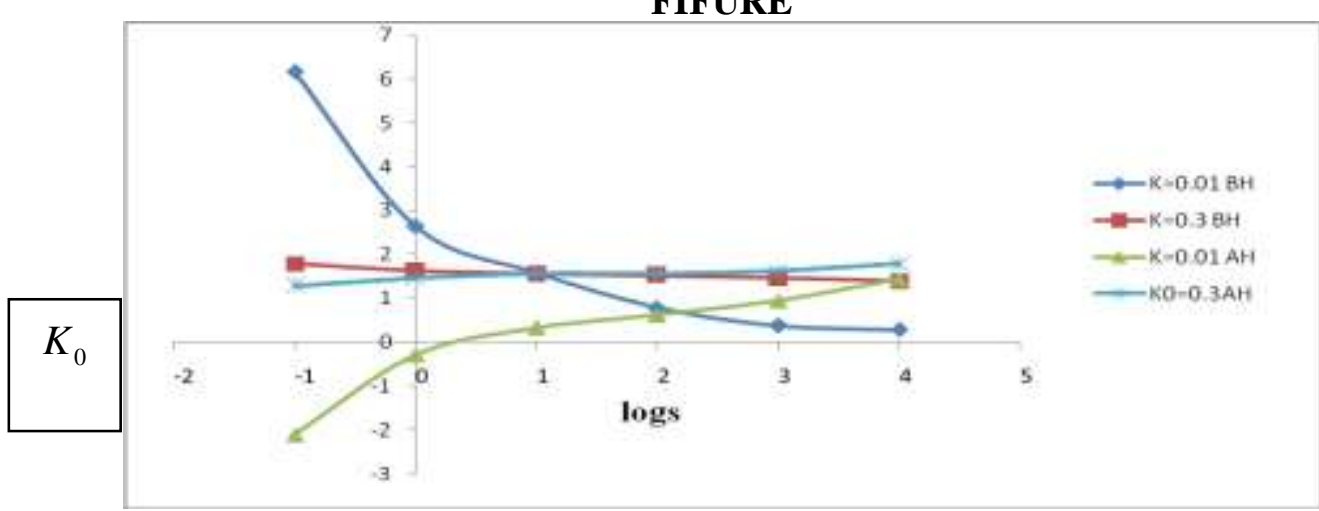




\section{Conclusion}

High magnetic field favours convective heat transfer whereas absence of magnetic field favours conductive heat transfer and reverse phenomenon could be observed if it is heated from above. It has been observed that a magnetic fluid in high magnetic field favours convective heat transfer and a non-magnetic fluid favours conductive heat transfer and reverse phenomenon could be observed if it is heated from above. It is clear That the differential equation is applied on similar pattern both in porous and non porous medium where as the stability pattern is subject to change with porosity and magnetic effect.

\section{References}

[1]. Baily R.L (1983): Lessor known applications of ferrofluids:- J.M.M.M.,vol.39,pp.178-182.

[2]. Berkovskii B.M., Medvedev V.F. and Krakov M.S(1993): Magnetic fluids-engineering applications-oxford: oxford science publications.

[3]. Chandrasekher S.(1961): Hydrodynamic and stability-oxford:Clarendon.

[4]. Finlayson B.A. (1970): Convective instability of ferromagnetic fluids -Journal of Fluid mech., Vol.40,pp.753-767.

[5]. E.R.Benton(1966). On the flow due to a rotating disk- Journal of Fluid mech. 24(4),pp.781-800.

[6]. H.Schlichting (1960), Boundary Layer Theory, McGraw-Hill Book company, New York..

[7]. H.A.Attia (2009),Steady flow over a rotating disk in porous medium with heat transfer. Non-Linear analysis modelling and control.14(1)pp.21-26.

[8]. J.L.Newringer, R.E.Rosensweig(1964),Magnetic fluids, Physics of fluids , 1927

[9]. M.I.Shliomis(2004), Ferrofluids as thermal ratchets. Physical Review Letters,92(18),188901.

[10]. P.D.S. Verma, M.Singh (1981),Magnetic fluid flow through porous annulus. Int.J.Non-linear Mechanics, 16(3/4),pp.371-378.

[11]. W.G.Cochran(1934), The flow due to a rotating disk. Proc.camb.Phil.Sot.30,pp.365-375. 